\title{
Control of angiogenesis by VEGF and endostatin-encapsulated protein microcrystals and inhibition of tumor angiogenesis
}

\author{
Goichi Matsumoto $^{a}$, Rie Hirohata ${ }^{b}$, Kousuke Hayashi $^{b}$, Yoko Sugimoto ${ }^{b}, E^{1 j i}$ Kotani ${ }^{b}$, \\ Junji Shimabukuro ${ }^{b}$, Tomoko Hirano ${ }^{c}$, Yumiko Nakajima ${ }^{d}$, Shin Kawamata ${ }^{e}$, \\ Hajime Mori $^{\mathrm{b}, \mathrm{f}, \boldsymbol{t}}$
}

aDivision of Oral Surgery, Yokohama Clinical Education Center of Kanagawa Dental University, 3-31-6 Tsuruya-cho, Kanagawa-ku, Yokohama 221-0835 Japan

${ }^{b}$ Department of Applied Biology, Kyoto Institute of Technology, Matsugasaki, Sakyo-ku, Kyoto 606-8585, Japan

${ }^{\circ}$ Venture Laboratory, Kyoto Institute of Technology, Matsugasaki, Sakyo-ku, Kyoto 606-8585, Japan

${ }^{\mathrm{d}}$ Functional Genomics Group, COMB, Tropical Biosphere Research Center, University of the Ryukyus, Okinawa, Japan

${ }^{\mathrm{e}}$ Basic Research Group for Regenerative Medicine, Foundation for Biomedical Research and Innovation, TRI308, 1-5-4 Minatojima-Minamimachi, Chuo-ku, Kobe 650-0043, Japan

IInsect Biomedical Research Center, Kyoto Institute of Technology. Matsugasaki, Sakyo-ku, Kyoto 606-8585, Japan

Key Words: VEGF, endostatin, polyhedra, angiogenesis, antiangiogenesis

${ }^{*}$ Corresponding author: Insect Biomedical Research Center, Kyoto Institute of Technology. Matsugasaki, Sakyo-ku, Kyoto 606-8585, Japan. Tel./fax: +81-75-724-7776.

E-mail address: hmori@kit.ac.jp 
Running title: Control of angiogenesis by VEGF and endostatin microcrystals 


\begin{abstract}
Encapsulation of cytokines within protein microcrystals (polyhedra) is a promising approach for the stabilization and delivery of therapeutic proteins. Here, we investigate the influence of vascular endothelial growth factor (VEGF) microcrystals and endostatin microcrystals on angiogenesis. VEGF was successfully encapsulated into microcrystals derived from insect cypovirus with overexpression of protein disulfide bond isomerase. VEGF microcrystals were observed to increase the phosphorylation of $\mathrm{p} 42 / \mathrm{p} 44$ MAP kinase and to stimulate the proliferation, migration, and network and tube formation of human umbilical vein endothelial cells (HUVECs). Endostatin was also successfully encapsulated into microcrystals. Endostatin microcrystals showed antiangiogenesis activities and inhibited the migration, and network and tube formation of HUVECs. Local administration of endostatin microcrystals in mice inhibited both angiogenesis and tumor growth with clear significant differences between treatment and control groups. Endostatin microcrystals only affected angiogenesis, but had no significant effect on lymphangiogenesis compared to controls. Local therapy using endostatin microcrystals offers a potential approach to achieve sustained therapeutic release of antiangiogenic molecules for cancer treatment.
\end{abstract}




\section{Introduction}

Cypovirus (CPV) infections result in the production of significant amounts of protein microcrystals (occlusion bodies) termed polyhedra. Virus particles become occluded within the polyhedra hence polyhedra are often referred to as occlusion bodies [1, 2]. Polyhedra are the main vectors by which virus particles transfer from insect to insect and are the main agents of survival of the virus from one insect generation to the next. Polyhedra stabilize virions thereby allowing them to remain viable for long periods in the environment $[3,4]$. We have successfully developed a system to immobilize foreign proteins into insect virus occlusion bodies [5]. We found that the virion outer capsid protein, VP3, is implicated in the occlusion of viral particles into Bombyx mori CPV (BmCPV) polyhedra, and identified that the N-terminal VP3 domain (VP3 immobilization signal) was responsible for association and incorporation of foreign proteins into BmCPV polyhedra $[5,6]$. The atomic structure of BmCPV polyhedra revealed that the polyhedrin $\mathrm{H} 1$ helix at the $\mathrm{N}$-terminus of the molecule is a useful tag for incorporating foreign proteins into polyhedra such as the VP3 immobilization signal $[4,7]$. In our new baculovirus expression vector system, the immobilization signal-containing foreign proteins expressed under the control of baculovirus polyhedrin promoter are occluded into BmCPV polyhedra consisting of crystallized polyhedrin expressed by another recombinant baculovirus containing BmCPV polyhedrin gene.

We have previously reported the encapsulation of fibroblast growth factor-2 (FGF-2) and FGF-7, epidermal growth factor (EGF), leukemia inhibitory factor, and secreted frizzled-related protein 4 into polyhedra [7-10]. These cytokines have biological activities as monomers. The vascular endothelial growth factor (VEGF) family plays an integral role in angiogenesis, lymphangiogenesis, and vasculogenesis $[11,12]$. The VEGF family currently comprises seven members (VEGF-A, VEGF-B, VEGF-C, VEGF-D, VEGF-E, VEGF-F, and placental growth factor). All members have a common VEGF homology domain. The core region is composed of a cystine knot motif, with eight invariant cysteine residues involved in inter- and intramolecular disulfide bonds at one end of a conserved central four-stranded $\beta$-sheet within each monomer, which 
dimerize in an antiparallel, side-by-side orientation [11-14]. VEGF-A is a 34- to 42-kDa, dimeric, disulfide-bound glycoprotein. VEGF-A exists in at least seven homodimeric isoforms where each subunit consists of $121,145,148,165,183,189$, or 206 amino acids [11, 12]. Before the encapsulation of VEGF-A into polyhedra, overexpression of protein disulfide bond isomerase (PDI) is required to obtain biologically active VEGF-encapsulated polyhedra.

Endostatin, a 20-kDa C-terminal fragment digested from collagen XVIII, inhibits the proliferation and migration of endothelial cells, and induces apoptosis, leading to repression of growth of a wide variety of tumors with neovascularization $[15,16]$. The apoptotic effect of endostatin is associated with decreased levels of anti-apoptotic molecules [17]. Recently, Zhuo et al. reported that endostatin directly inhibits lymphatic endothelial cells (LEC) and lymphangiogenic vessels via the cell surface receptor nucleolin on LEC which inhibits lymphatic metastasis [18].

In this study VEGF and endostatin were encapsulated into polyhedra and the effect of both VEGF and endostatin polyhedra on angiogenesis in cell culture and in vivo was investigated.

\section{Materials and methods}

\subsection{Medium}

Growth medium: EGM-2 (LONZA) containing 2\% fetal bovine serum (FBS), EGF, FGF-2, VEGF, $\mathrm{R}^{3}$-IGF-1, hydrocortisone, ascorbic acid, heparin, GA-100 (gentamicin, amphotericin-B). Assay medium: EGM-2 containing 0.2\% FBS, hydrocortisone, ascorbic acid, heparin, GA-1000. Starvation medium: $\alpha$ MEM (WAKO).

\subsection{Construction of VEGF and endostatin transfer vector}

Recombinant VEGF-A baculovirus transfer vectors were generated using GATEWAY $^{\circledR}$ cloning technology (Invitrogen). The VEGF-A gene (189 aa) fused to DNA encoding either VP3 immobilization signal at $\mathrm{N}$-terminal or $\mathrm{C}$-terminal was amplified by polymerase chain reaction (PCR) using primer sets containing attB1 and attB2 sequences below: 
5'-GGGGACAAGTTTGTACAAAAAAGCAGGCTTAATGGCACCCACGACAGAAGGAGAGC-3'

and

reverse

primers

are

5'-GGGGACCACTTTGTACAAGAAAGCTGGGTATCACCGCCTTGGCTTGTCACATCTGCA-3'

for N-terminal VP3 or

5'-GGGGACCACTTTGTACAAGAAAGCTGGGTACCGCCTTGGCTTGTCACATCTGCA-3' for C-terminal VP3. The resulting attB-flanked PCR products were cloned into a donor vector (pDONR221) to construct entry vectors by BP reactions of GATEWAY ${ }^{\circledR}$ system. The open reading frame cloned between the att 1 and att 22 sites in the entry vectors was transferred to destination vectors ( $\mathrm{pDEST}-\mathrm{N}-\mathrm{VP3}$ or pDEST-C-VP3) via LR Clonase ${ }^{\mathrm{TM}}$ reactions resulting in production of the transfer vectors encoding VEGF-A fused with VP3 tag (pVP3/VEGF and pVEGF/VP3).

DNA fragment encoding C-terminal region (183 aa) of collagen XVIII was fused with H1 or VP3 immobilization signal and also amplified by PCR using primer sets containing attB1 and attB2 sequences below:

the forward primer is 5'-GGGGACAAGTTTGTACAAAAAAGCAGGCTTAATGCACAGCCACCGCGACTTCCAGC -3' $\begin{array}{lll}\text { and } & \text { reverse } & \text { primers }\end{array}$ 5'-GGGGACCACTTTGTACAAGAAAGCTGGGTACTACTTGGAGGCAGTCATGAAGCTGTT-3' for $\quad \mathrm{H} 1$ signal at $\quad \mathrm{N}$-terminal 5'-GGGGACCACTTTGTACAAGAAAGCTGGGTACTTGGAGGCAGTCATGAAGCTGTT-3' for VP3 signal at C-terminal. The resulting attB-flanked PCR products were also cloned into pDONR221 to construct entry vectors. The open reading frame cloned between the attL1 and attL2 sites in the entry vectors was transferred to destination vectors (pDEST-N-H1 or pDEST-C-VP3) resulting in production of the transfer vectors encoding endostatin fused with $\mathrm{H} 1$ or VP3 tags (pH1/Endo and pEndo/VP3). 


\subsection{Production of VEGF and endostatin polyhedra}

The transfer vectors were co-transfected into Spodoptera frugiperda IPLB-Sf21-AE (Sf21) cells with linearized Baculogold Baculovirus DNA (BD Pharmingen). After incubation for 5 days at $27^{\circ} \mathrm{C}$, recombinant baculoviruses expressing VEGF-A and endostatin were harvested and stored at $4 \stackrel{\circ}{\circ} \mathrm{C}$. To incorporate VEGF-A into polyhedra (VEGF polyhedra), immobilization of VEGF-A using VP3 tag was accomplished by dual infection of the recombinant baculoviruses that express the VEGF-A and a recombinant baculovirus AcCP-H expressing BmCPV polyhedrin or AcCP-H/PDI expressing BmCPV polyhedrin and PDI, in which BmCPV polyhedrin and PDI were expressed under the control of the baculovirus polyhedrin promoter and p10 promoter, respectively. Endostatin polyhedra were also made by dual infections with recombinant baculoviruses expressing endostatin with $\mathrm{H} 1$ tag (AcH1/Endo) or VP3 tag (AcEndo/VP3) and AcCP-H, resulting in H1/Endo polyhedra and Endo/VP3 polyhedra. To generate empty polyhedra, Sf cells were inoculated with recombinant baculovirus AcCP-H or AcCP-H29/PDI. The infected cells were cultured for 10 days at $27^{\circ} \mathrm{C}$ and then the cells were harvested in a conical tube by centrifugation. The cell pellet was resuspended in phosphate-buffered saline (PBS; pH 7.2) and treated with an ultrasonic homogenizer at $6 \%$ power for $30 \mathrm{sec}$. The cell homogenate was centrifuged at $1500 \times \mathrm{g}$ at $4{ }^{\circ} \mathrm{C}$ and the supernatant was removed. These treatments were repeated and the purification was complete. The polyhedron suspension was adjusted to the same density $\left(5 \times 10^{4}\right.$ numbers $/ \mu \mathrm{l})$ and stored at $4{ }^{\circ} \mathrm{C}$ in distilled water containing $100 \mathrm{units} / \mathrm{ml}$ penicillin and $100 \mu \mathrm{g} / \mathrm{ml}$ streptomycin.

\subsection{Phosphorylation of p44/p42 mitogen-activated protein kinase (MAP kinase)} Human umbilical vein endothelial cells (HUVECs) were plated into a 6 -well plate at a density of 1 $\times 10^{5}$ cells per well and cultured to confluence in the growth medium. Cells were washed with PBS and then incubated with the starvation medium for 6 hours to synchronize cell cycles. The cultures were then treated with rhVEGF and empty or VEGF polyhedra. HUVECs were washed with PBS 
twice and dissolved in $100 \mu \mathrm{l}$ of $1 \times$ SDS sample buffer containing $50 \mathrm{mM}$ Tris- $\mathrm{HCl}(\mathrm{pH} 6.8), 2 \%$ SDS, $50 \mathrm{mM}$ dithiothreitol, $0.1 \%$ bromophenol blue, and $10 \%$ glycerol. Cell lysates were harvested, sonicated, and cleaned by centrifugation. Samples were electrophoresed on $12 \%$ SDS-polyacrylamide gels and transferred to nitrocellulose membranes (Bio-Rad). After preincubation with blocking buffer containing $5 \%$ nonfat milk, $0.1 \%$ Tween $20,25 \mathrm{mM}$ Tris- $\mathrm{HCl}(\mathrm{pH}$ 7.6), $150 \mathrm{mM} \mathrm{NaCl}$, the membranes were incubated with either p44/42 MAP Kinase Antibody or Phospho-p44/42 MAP kinase (Thr202/Tyr204) Antibody followed by horseradish peroxidase (HRP)-conjugated anti-rabbit IgG antibody (New England Biolabs). Peroxidase activity was detected and visualized by ECL Western Blotting Detection Reagents (GE Healthcare).

\subsection{Proliferation assay of HUVECs}

The proliferation of HUVECs was measured by Cell Counting Kit-8 using WST-8 (DOJINDO). Cells were seeded into 96-well plates at a density of $3 \times 10^{3}$ cells/well. After incubation in the assay medium containing rhVEGF and empty or VEGF polyhedra for 3 days at $37^{\circ} \mathrm{C}$ under $5 \%$ $\mathrm{CO}_{2}$, Cell Counting Kit-8 solution was added to the cultures and incubated for 4 hours at $37^{\circ} \mathrm{C}$ under $5 \% \mathrm{CO}_{2}$, according to the manufacturer's instructions. Absorbance at $450 \mathrm{~nm}$ was determined with a microplate reader Model 680 (Bio-Rad).

\subsection{In vitro scratch assay}

We used the in vitro scratch assay to assess the activity of VEGF polyhedra and endostatin polyhedra on migration of HUVECs. HUVECs were seeded in 24 -well plates $\left(8 \times 10^{3} /\right.$ well) with the growth medium and cultured to confluence in the growth medium. The cells were scraped away vertically 24 hours later by pipette tip. Each well was washed twice with PBS to remove debris, and then further incubated for 18 hours in serum-free EGM-2 medium with rhVEGF or polyhedra (empty, VEGF, endostatin). Endostatin polyhedra were assayed in the presence of 10 $\mathrm{ng} / \mathrm{ml}$ rhVEGF. The distances between the 2 edges of the scratch were photographed on each 
well using an inverted microscope.

\subsection{Network formation of HUVECs}

Subconfluent culture of HUVECs were washed with PBS and suspended in the starvation medium to synchronize cell cycles. After rhVEGF or polyhedra (empty, VEGF, endostatin) were mixed with ECMatrix (CHEMICON) solution, $50 \mu \mathrm{l}$ of the aliquot were added to each well of the 96 -well plate and the plate was incubated for 1 hour at $37^{\circ} \mathrm{C}$ to allow the matrix solution to solidify. To assay endostatin polyhedra, ECMatrix solution containing rhVEGF $(10 \mathrm{ng} / \mathrm{ml})$ was used. HUVECs $(1 \times$ $10^{4} /$ well) were seeded onto presolidified ECMatrix. After incubation for 18 hours at $37^{\circ} \mathrm{C}$ under $5 \%$ $\mathrm{CO}_{2}, 50 \mu \mathrm{l}$ of Calcein AM (BD Biosciences) solution was added to each well and plates were incubated at $37^{\circ} \mathrm{C}$ under $5 \% \mathrm{CO}_{2}$ for 30 minutes. Total tube length of each well was measured using an image analyzer (ESPEC TECHNO CORP).

\subsection{Tube formation of HUVECs}

Normal human dermal fibroblast cells $\left(2 \times 10^{4} /\right.$ well $)$ were seeded onto a collagen-coated 24 -well plate and incubated with $500 \mu \mathrm{L}$ of Medium 106 supplemented with Low Serum Growth Supplement containing 2\% FBS, EGF, FGF-2, hydrocortisone, heparin (Life Technologies) for 24 hours at $37^{\circ} \mathrm{C}$ under $5 \% \mathrm{CO}_{2}$. The medium was replaced with the starvation medium and then rhVEGF or polyhedra (empty, VEGF, endostatin) were added to each well followed by incubation for 1 hour at $37^{\circ} \mathrm{C}$ under $5 \% \mathrm{CO}_{2}$. Endostatin polyhedra were assayed in the presence of $10 \mathrm{ng} / \mathrm{ml}$ rhVEGF. HUVECs $\left(2 \times 10^{4} /\right.$ well $)$ were seeded into each well and incubated with the assay medium for 11 days at $37^{\circ} \mathrm{C}$ under $5 \% \mathrm{CO}_{2}$. The assay medium with or without $\mathrm{rhVEGF}(10 \mathrm{ng} / \mathrm{ml})$ was used for wells of rhVEGF or polyhedra, respectively, at each 2 days medium change. Cells were washed with PBS and fixed with cold $70 \%$ ethanol. After 30 min, ethanol was discarded and cells were incubated with $1 \%$ BSA. Fixed cells were reacted with mouse anti-human CD31 antibody and then goat anti-mouse IgG AP conjugate antibody. Tube formation of HUVEC was 
detected by addition of substrate BCIP/NBT.

\subsection{Tumor cell line and cell cultures}

The SCC-VII tumors were cutaneous mouse squamous cell carcinomas that spontaneously arose in the $\mathrm{C} 3 \mathrm{H} / \mathrm{He}$ mouse strain [19]. SCC-VII cells were inoculated subcutaneously into $\mathrm{C} 3 \mathrm{H} / \mathrm{He}$ mice. We cultured SCC-VII cells in RPMI 1640 medium supplemented with 10\% heat-inactivated FCS at $37^{\circ} \mathrm{C}$ in an incubator with $5 \% \mathrm{CO}_{2}$.

\subsection{Tumor model and treatment}

Our animal studies conformed to the Kanagawa Dental University guidelines for research animal care and were approved by ethical committees of Kanagawa Dental University. Subcutaneous injection of $5 \times 10^{5}$ SCC-VII cells in $100 \mu$ of PBS into the backs of $\mathrm{C} 3 \mathrm{H} / \mathrm{He}$ mice (Japan SLC, Shizuoka, Japan) aged 6 weeks generated back tumors. Tumor volume was estimated using the formula: tumor volume $\left(\mathrm{mm}^{3}\right)=$ length $(\mathrm{mm}) \times$ cross-section $(\mathrm{mm})^{2} \times 1 / 2$. Two weeks later, when the tumors had grown to $100-200 \mathrm{~mm}^{3}$, we randomly divided the mice into 4 treatment groups: $5 \mathrm{x}$ $10^{6} \mathrm{H} 1 /$ Endo polyhedra with $100 \mu \mathrm{PBS} ; 5 \times 10^{6}$ Endo/VP3 polyhedra with $100 \mu \mathrm{l}$ PBS; $5 \times 10^{6}$ empty polyhedra with $100 \mu \mathrm{l}$ PBS; and no treatment as a control. On the $2^{\text {nd }}$ and $4^{\text {th }}$ week after the inoculation of tumor cells, we administered each polyhedron by intratumoral injection. The control group was without treatment. After SCC-VII tumor-cell inoculation, tumor volume was measured at 6 weeks. Each group contained 4 mice.

\subsection{Immunohistochemical analysis}

Implanted-tumor specimens from mice were embedded in OCT compound, quickly frozen in dry ice, and stored at $-80{ }^{\circ} \mathrm{C}$ for immunohistochemical staining. To analyze tumor angiogenesis and lymphangiogenesis, we performed double immunofluorescence staining of excised tumors for the vascular marker CD31 and for the lymphatic-specific marker LYVE-1. Frozen sections of $6 \mu \mathrm{m}$ 
thickness were stained using a rat anti-mouse CD31 mAb (clone MEC13.3, Pharmingen, CA) for 60 min at RT and then rinsed 3 times. Sections were then stained with anti-rat antibody conjugated with Alexa Fluor 488 (Invitrogen, CA) for 60 min at RT. After staining with CD31, the sections were rinsed 3 times in PBS and incubated a second time with anti-LYVE-1 polyclonal Ab (Abcam, Cambridge, UK) for 60 min at RT and then rinsed 3 times. Sections were then stained with anti-rabbit antibody conjugated with Alexa Fluor 546 (Invitrogen, CA) for 60 min at RT. Cell nuclei were counterstained with DAPI (InnoGenex, CA). Slides were then mounted with a fluorescent mounting medium (DAKO, CA). Images were obtained on a Zeiss microscope and analyzed using Axiolmager software (Carl Zeiss, NY). Microvessel density and lymphatic microvessel density in the tumors were determined after immunostaining with antibodies against CD31- and LYVE-1- positive vessels, which were counted microscopically under x100 magnification from 3 areas of the highest vascular density per section.

\section{Results}

\subsection{Proliferation, migration, network and tube formation of HUVECs by VEGF polyhedra}

VEGF is secreted as a cysteine-linked dimer and has two binding sites at opposite poles through which it binds VEGF receptors (VEGFRs). VEGFRs are tyrosine kinases found on the surface of endothelial and other cells. VEGF-A tagged with VP3 at N-terminus or C-terminus was incorporated into polyhedra with or without the expression of PDI and biological activities of these polyhedra were studied. To examine activation of the downstream signaling pathway by polyhedra encapsulating VEGF-A, phosphorylation of p44/p42 MAP kinase was analyzed in HUVECs. Phosphorylated forms of p44 and p42 MAP kinase were detected in HUVECs incubated with rhVEGF as well as VEGF/VP3+PDI polyhedra, in which VEGF-A fused with VP3 at C-terminus and co-expressed with PDI was encapsulated (Fig. 1A). In contrast, phosphorylation was not or was poorly detected in the cells incubated with empty polyhedra, nor was detection observed for 
other polyhedra in which VEGF-A was encapsulated by VP3 at N-terminus or without PDI expression. The VEGFRs require dimerization to be active. Signaling is initiated when VEGF binds first to one of the receptors, resulting in translocation of the second receptor for dimerization. As it was considered that firstly dimerization of VEGF-A was caused by PDI and then VEGF-A molecules were incorporated into polyhedra, we used these polyhedra as VEGF polyhedra for assay of proliferation, migration, and network and tube formation of HUVECs.

We have previously reported similar results regarding encapsulation of bone morphogenetic protein 2 (BMP-2) into polyhedra [20]. Native BMP-2 protein consists of two polypeptide chains linked by an S-S bond between Cys78 of each chain. Co-expression of PDI was needed to obtain biologically active BMP-2 polyhedra during encapsulation into polyhedra. BMP-2 expressed in Escherichia coli is accumulated in inclusion bodies and the first step of preparation is isolation of inclusion bodies from E. coli cells. Secondly, denaturation of inclusion bodies and purification are necessary. Finally, subsequent refolding of BMP-2 is required to attain the biologically active homodimeric form [21]. VEGF is usually expressed in E. coli cells and purified by similar steps $[22,23]$. Our data show that the isolation and denaturation of inclusion bodies and subsequent refolding into the biologically active homodimeric form are unnecessary when cytokines for encapsulated into polyhedra.

Proliferation of HUVECs in the presence of VEGF polyhedra showed a dose-response curve (Fig. 1B). VEGF activity from $8.75 \times 10^{4}$ numbers of VEGF polyhedra was estimated to be equivalent to the activity of $1 \mathrm{ng}$ rhVEGF. Endothelial cell migration is a necessary step of angiogenesis. The effect of VEGF polyhedra on HUVECs migration was determined using the scratch assay. As shown in Figure 2, cell migration was stimulated by VEGF polyhedra. The formation of three-dimensional capillary-like structures by HUVECs is well-established in in vitro angiogenesis assays. HUVECs were seeded on Matrigel substrate and dispersed evenly throughout the culture. Thereafter, cells spontaneously aligned to form a network of capillary-like structures in the presence of rhVEGF or VEGF polyhedra (Fig. 3A, B). The formation of tube-like 
structures is an essential step in angiogenesis. Therefore, HUVEC angiogenesis was also observed using the tube formation assay. As shown in Figure 3C, capillary tube structures were formed by rhVEGF or VEGF polyhedra after HUVECs were placed in the wells, indicating that VEGF polyhedra can stimulate angiogenesis.

\subsection{Antiangiogenic activities of endostatin polyhedra}

We studied the antiangiogenic activities of endostatin polyhedra. Endostatin fused with H1 or VP3 tags was incorporated into polyhedra (H1/Endo polyhedra and Endo/VP3 polyhedra, respectively). The antimigratory effect of both endostatin polyhedra was observed by the inhibition of migration of HUVECs (Fig. 2). Addition of endostatin polyhedra to the culture media significantly inhibited the network and tube formation of HUVECs (Fig. 3), indicating that endostatin polyhedra had potent anti-endothelial cell activity.

\subsection{Inhibition of tumor growth by endostatin polyhedra injections}

Approximately 2 weeks later, when the tumors had grown to $100-200 \mathrm{~mm}^{3}$, the mice were randomly assigned to groups and therapy was initiated. H1/Endo polyhedra, Endo/VP3 polyhedra and empty polyhedra were administrated at 2 and 4 weeks after tumor-cell inoculation. At 6 weeks post-inoculation, the tumor volumes of endostatin polyhedra groups (H1/Endo polyhedra and Endo/VP3 polyhedra) were lower than those of control or empty polyhedra groups (Fig.4). We concluded that local therapy with endostatin encapsulated into polyhedra as a carrier efficiently inhibited tumor growth in vivo.

To identify the mechanism of tumor growth inhibition, we examined angiogenesis of tumor tissues by immunohistochemical staining. Angiogenesis within the tumor tissues was determined by directly counting the number of $\mathrm{CD} 31^{+}$cells on microvessels. The most highly vascularized areas of each tumor were identified and $\mathrm{CD} 31^{+}$vessels were counted in at least five fields (magnification: x100). Angiogenesis was inhibited in the treatment with $\mathrm{H} 1 /$ Endo polyhedra or 
Endo/VP3 polyhedra, and there was a significant difference compared to the number of vessels counted for the control and empty polyhedra groups. There was a pronounced inhibition of angiogenesis in tumors treated with the H1/Endo polyhedra or Endo/VP3 polyhedra (Fig. 5B). However for lymphatic vessel assay, no statistically significant differences in lymphatic vessel densities were observed in all experimental groups compared to controls (Fig. 5C). To conclude, endostatin polyhedra were effective in the blood vessel assay but were not effective in the lymphatic vessel assay. Endostatin polyhedra therefore may have anti-angiogenic effect but likely do not have anti-lymphangiogenic effect.

\section{Discussion}

Cytokines are not diffusible and bind to the cell surface or surrounding extracellular matrix (ECM) $[24,25]$. FGF-2 binds to heparan sulfate in ECM and ECM-bound FGF-2 is released by exposure to heparin or heparan sulfate [26]. FGF-2 released from ECM is biologically active and stimulates cell proliferation and DNA synthesis in vascular endothelial cells and 3T3 fibroblasts. VEGF and endostatin have heparin-binding domains which are critical in angiogenesis and antiangiogenesis, respectively [27-29]. Release of VEGF and endostatin from ECM and their activation is triggered by the binding of heparin. Such a signaling cue originating from the extracellular microenvironment is also essential for cellular processes including proliferation, differentiation, migration and apoptosis. For in vivo study of interactions between cytokines and target cells or tissues, reservoirs or slow-release agents are necessary for sustained release of active cytokines. If the cytokines are unstable molecules, such a reservoir might be more important for in vivo study.

Dr. Folkman observed that endostatin has potent antiangiogenesis activity and endostatin was effective in inhibiting tumor growth in several preclinical studies, however the response in clinical trials was less impressive. It was difficult for other laboratories to reproduce these experiments. There are some practical problems in expression, refolding, bioavailability, short half-life, purification and stock of endostatin due to its instability [30]. There are various 
methods by which to transfect endostatin cDNA into cells. One way is to use a viral vector to incorporate the gene or gene fragment into the cells [31, 32]. While this method allows effective and reliable supply of endostatin in the cell, there is an unavoidable risk that the virus may cause harm to the host.

To mitigate these problems, we have genetically modified endostatin to improve its biological activity. The modified cypovirus polyhedra can function as nano- or micro-containers stabilizing incorporated proteins in a functional form [2]. One application is as a mimic of ECM whereby cytokines including growth factors can be expressed in the polyhedra to mimic their natural structural scaffolds such as collagen, fibronectin and heparan sulfate. In the ECM, enhanced stability and activity of the embedded cytokines results in cell proliferation and differentiation regulated in time and space $[24,25]$. Such perspectives can drive active research on the applications of polyhedra in stem cell research, tissue engineering and regenerative medicine. Another application is a novel tool for "proof of concept". Unstable cytokine molecules are rapidly degraded and the biological activities are lost. However, purification of cytokines becomes unnecessary when encapsulating within polyhedra. We would like to propose the use of polyhedra for elucidation or identification of unknown functions of cytokines.

Tumor growth and metastatic dissemination depend on the formation of new tumor microvessels. The process of tumor angiogenesis is a balance between proangiogenic and antiangiogenic factors. The use of antiangiogenic agents to restore this balance represents a useful approach in cancer treatment. Inhibition of tumor angiogenesis by antiangiogenic agents for anticancer therapy is widely accepted.

Generally, the lymphatic system plays important roles in maintaining fluid balance in inflammation and cancer [33, 34]. In cancer, lymphangiogenesis, the sprouting of new lymphatic vessels from the preexisting lymphatic system, may provide a way to facilitate the dissemination of tumor cells to sentinel lymph nodes and other distant organs [34, 35]. Recently, several researchers reported that endostatin inhibits lymph node metastasis via down-regulation of 
VEGF-C, either by tumor cells or by mast cells in the tumor micro-environment $[36,37$. Interestingly, endostatin directly acts on lymphangiogenic endothelial cells via cell surface receptor nucleolin on LECs and inhibits tumor lymphangiogenesis [18]. Here, we did not detect the effects of endostatin on tumor lymphangiogenesis and lymph node metastasis. These discrepancies may be due to the use of different tumor models. SCC-VII tumors are cutaneous mouse squamous cell carcinomas that spontaneously arise in the $\mathrm{C} 3 \mathrm{H} / \mathrm{He}$ mouse strain and do not metastasize [38].

Antiangiogenic cancer therapy with antiangiogenic agents requires the peptide to be administered regularly or daily over a prolonged period, implying that large quantities of the therapeutic agent are needed. These functional antiangiogenic proteins are expensive to produce, and frequently there are technical problems related to physical properties and purity. Moreover, some therapeutic agents that could otherwise provide effective antiangiogenic cancer therapy have biological half-lives too short to sustain tumor regression. All of the above factors are obstacles for effective therapies using exogenously injected antiangiogenic agents to inhibit tumor angiogenesis. Polyhedral microcrystals represent an attractive and promising alternative method to deliver antiangiogenic agents. We reported that polyhedron microcrystals can remain in the connective tissue over five weeks after implantation [20]. Consequently, therapy using polyhedral microcrystals has the potential to produce the antiangiogenic agent in high concentrations in a tumor area over a sustained period, thereby avoiding the problems encountered with long-term administration of recombinant proteins.

\section{Conclusion}

VEGF fused with VP3 tag at the C-terminus was co-expressed with PDI and encapsulated into insect virus-derived protein microcrystals, polyhedra. The polyhedra stimulated phosphorylation of P44/P42 MAP kinase of HUVECs. VEGF polyhedral stimulated angiogenesis as revealed by assays of proliferation, migration, and network and tube formation of HUVECs. Endostatin fused 


\section{Acknowledgments}

This work was supported in part by A-STEP exploratory research AS232Z00847F from JST, Grants-in-Aid for Scientific Research (A) 22241052 and (C) 90237131 from JSPS, and Regional Consortium Research Development Work and Regional Innovation Creation R\&D Programs of Kansai Bureau of Economy, Trade and Industry of Japan. 


\section{References}

[1] Belloncik S, Mori H. Cypovirus. In: Miller LK, Ball LA, editors. The insect viruses. New York: Plenum Press; 1998. p. 337-69.

[2] Mori H, Metcalf, P. Cypoviruses. In: Asgari S, Johnson, K. N., editor. Insect Virology: Caister Academic Press; 2010. p. 307-24.

[3] Rohrmann GF. Polyhedrin structure. J Gen Virol. 1986;67 ( Pt 8):1499-513.

[4] Coulibaly F, Chiu E, Ikeda K, Gutmann S, Haebel PW, Schulze-Briese C, et al. The molecular organization of cypovirus polyhedra. Nature. 2007;446(7131):97-101.

[5] Ikeda K, Nagaoka S, Winkler S, Kotani K, Yagi H, Nakanishi K, et al. Molecular characterization of Bombyx mori cytoplasmic polyhedrosis virus genome segment 4. J Virol. 2001;75(2):988-95.

[6] Ikeda K, Nakazawa H, Shimo-Oka A, Ishio K, Miyata S, Hosokawa Y, et al. Immobilization of diverse foreign proteins in viral polyhedra and potential application for protein microarrays. Proteomics. 2006;6(1):54-66.

[7] Ijiri H, Coulibaly F, Nishimura G, Nakai D, Chiu E, Takenaka C, et al. Structure-based targeting of bioactive proteins into cypovirus polyhedra and application to immobilized cytokines for mammalian cell culture. Biomaterials. 2009;30(26):4297-308.

[8] Mori H, Shukunami C, Furuyama A, Notsu H, Nishizaki Y, Hiraki Y. Immobilization of bioactive fibroblast growth factor-2 into cubic proteinousmicrocrystals (Bombyx mori cypovirus polyhedra) that are insoluble in a physiological cellular environment. J Biol Chem. 2007;282(23):17289-96.

[9] Nishishita N, ljiri H, Takenaka C, Kobayashi K, Goto K, Kotani E, et al. The use of leukemia inhibitory factor immobilized on virus-derived polyhedra to support the proliferation of mouse embryonic and induced pluripotent stem cells. Biomaterials. 
$2011 ; 32(14): 3555-63$

[10] Matsushima K, Suyama T, Takenaka C, Nishishita N, Ikeda K, Ikada Y, et al. Secreted frizzled related protein 4 reduces fibrosis scar size and ameliorates cardiac function after ischemic injury. Tissue Eng Part A. 2010;16(11):3329-41.

[11] Hoeben A, Landuyt B, Highley MS, Wildiers H, Van Oosterom AT, De Bruijn EA. Vascular endothelial growth factor and angiogenesis. Pharmacol Rev. 2004;56(4):549-80.

[12] Ferrara N. Vascular endothelial growth factor: basic science and clinical progress. Endocr Rev. 2004;25(4):581-611.

[13] Neufeld G, Cohen T, Gengrinovitch S, Poltorak Z. Vascular endothelial growth factor (VEGF) and its receptors. FASEB J. 1999;13(1):9-22.

[14] Ortega N, Hutchings H, Plouet J. Signal relays in the VEGF system. Front Biosci. 1999;4:D141-52.

[15] O'Reilly MS, Boehm T, Shing Y, Fukai N, Vasios G, Lane WS, et al. Endostatin: an endogenous inhibitor of angiogenesis and tumor growth. Cell. 1997;88(2):277-85.

[16] Folkman J. Antiangiogenesis in cancer therapy--endostatin and its mechanisms of action. Exp Cell Res. 2006;312(5):594-607.

[17] Dixelius J, Larsson H, Sasaki T, Holmqvist K, Lu L, Engstrom A, et al. Endostatin-induced tyrosine kinase signaling through the Shb adaptor protein regulates endothelial cell apoptosis. Blood. 2000;95(11):3403-11.

[18] Zhuo W, Luo C, Wang X, Song X, Fu Y, Luo Y. Endostatin inhibits tumour lymphangiogenesis and lymphatic metastasis via cell surface nucleolin on lymphangiogenic endothelial cells. J Pathol. 2010;222(3):249-60.

[19] Chaplin DJ, Olive PL, Durand RE. Intermittent blood flow in a murine tumor: 
radiobiological effects. Cancer Res. 1987;47(2):597-601.

[20] Matsumoto G, Ueda T, Shimoyama J, ljiri H, Omi Y, Yube H, et al. Bone regeneration by polyhedral microcrystals from silkworm virus. Sci Rep. 2012;2:935.

[21] Sharapova NE, Kotnova AP, Galushkina ZM, Lavrova NV, Poletaeva NN, Tukhvatulin $A E$, et al. [Production of the recombinant human bone morphogenetic protein-2 in Escherichia coli and testing of its biological activity in vitro and in vivo]. Mol Biol (Mosk). 2010;44(6):1036-44.

[22] Heiring C, Muller YA. Folding screening assayed by proteolysis: application to various cystine deletion mutants of vascular endothelial growth factor. Protein Eng. $2001 ; 14(3): 183-8$.

[23] Seyedarabi A, Cheng L, Zachary I, Djordjevic S. Production of soluble human vascular endothelial growth factor VEGF-A165-heparin binding domain in Escherichia coli. PLoS One. 2013;8(2):e55690.

[24] Ramirez F, Rifkin DB. Cell signaling events: a view from the matrix. Matrix Biol. 2003;22(2):101-7.

[25] Sternlicht MD, Werb Z. How matrix metalloproteinases regulate cell behavior. Annu Rev Cell Dev Biol. 2001;17:463-516.

[26] Bashkin P, Doctrow S, Klagsbrun M, Svahn CM, Folkman J, Vlodavsky I. Basic fibroblast growth factor binds to subendothelial extracellular matrix and is released by heparitinase and heparin-like molecules. Biochemistry. 1989;28(4):1737-43.

[27] Fairbrother WJ, Champe MA, Christinger HW, Keyt BA, Starovasnik MA. Solution structure of the heparin-binding domain of vascular endothelial growth factor. Structure. $1998 ; 6(5): 637-48$.

[28] Javaherian K, Park SY, Pickl WF, LaMontagne KR, Sjin RT, Gillies S, et al. Laminin 
modulates morphogenic properties of the collagen XVIII endostatin domain. J Biol Chem. 2002;277(47):45211-8.

[29] Sasaki T, Larsson H, Kreuger J, Salmivirta M, Claesson-Welsh L, Lindahl U, et al. Structural basis and potential role of heparin/heparan sulfate binding to the angiogenesis inhibitor endostatin. EMBO J. 1999;18(22):6240-8.

[30] Fu Y, Tang H, Huang Y, Song N, Luo Y. Unraveling the mysteries of endostatin. IUBMB Life. 2009;61(6):613-26.

[31] Sun X, Krissansen GW, Fung PW, Xu S, Shi J, Man K, et al. Anti-angiogenic therapy subsequent to adeno-associated-virus-mediated immunotherapy eradicates Iymphomas that disseminate to the liver. Int J Cancer. 2005;113(4):670-7.

[32] Noro T, Miyake K, Suzuki-Miyake N, Igarashi T, Uchida E, Misawa T, et al. Adeno-associated viral vector-mediated expression of endostatin inhibits tumor growth and metastasis in an orthotropic pancreatic cancer model in hamsters. Cancer Res. 2004;64(20):7486-90.

[33] Swartz MA. The physiology of the lymphatic system. Adv Drug Deliv Rev. $2001 ; 50(1-2): 3-20$.

[34] Cao Y. Opinion: emerging mechanisms of tumour lymphangiogenesis and lymphatic metastasis. Nat Rev Cancer. 2005;5(9):735-43.

[35] Gao P, Zhou GY, Zhang QH, Su ZX, Zhang TG, Xiang L, et al. Lymphangiogenesis in gastric carcinoma correlates with prognosis. J Pathol. 2009;218(2):192-200.

[36] Fukumoto S, Morifuji M, Katakura Y, Ohishi M, Nakamura S. Endostatin inhibits lymph node metastasis by a down-regulation of the vascular endothelial growth factor $\mathrm{C}$ expression in tumor cells. Clin Exp Metastasis. 2005;22(1):31-8.

[37] Brideau G, Makinen MJ, Elamaa H, Tu H, Nilsson G, Alitalo K, et al. Endostatin 
1

2

overexpression inhibits lymphangiogenesis and lymph node metastasis in mice. Cancer Res. 2007;67(24):11528-35.

[38] Matsumoto G, Ohmi Y, Shindo J. Angiostatin gene therapy inhibits the growth of murine squamous cell carcinoma in vivo. Oral Oncol. 2001;37(4):369-78. 


\section{Legends to figures}

Fig. 1 Phosphorylation of p44/p42 MAP kinase and proliferation of HUVECs by polyhedra encapsulating VEGF-A. (A) HUVECs were plated into a 6-well plate at a density of $1 \times 10^{5}$ cells per well and cultured to confluence in the growth medium. Cells were starved in the starvation medium for 6 hours and then incubated with rhVEGF $(5 \mathrm{ng} / \mathrm{ml})$ and empty or VEGF polyhedra (2.5 $\times 10^{5}$ numbers/ml) for 30 min. VP3/VEGF and VEGF/VP3 show VEGF polyhedra in which VEGF was encapsulated into polyhedra by VP3 tag at $\mathrm{N}$ - or $\mathrm{C}$-terminus with or without PDI, respectively. Empty is CP-H polyhedra. The phosphorylated p44 (phospho-p44) and p42 (phospho-p42) MAP kinase were immunoblotted with Phospho-p44/p42 MAP Kinase Antibody and total amounts of p44/p42 were detected by p44/p42 MAP Kinase Antibody. (B) HUVECs were plated into a 96-well plate at a density of $3 \times 10^{3}$ cells per well and starved in the starvation medium for 6 hours. Each number $\left(0.2,0.5,1,2 \times 10^{5}\right)$ of VEGF polyhedra, $2 \times 10^{5}$ numbers of empty polyhedra and $2 \mathrm{ng}$ of rhVEGF in $200 \mu \mathrm{l}$ of the assay medium were added into wells. After 3 days, the proliferation of HUVECs was measured by Cell Counting Kit-8. The graph shows mean +/- S.E.

Fig. 2 Stimulation and inhibition of migration of HUVECs by VEGF and endostatin polyhedra. HUVECs were plated into a 24-well plate at a density of $8 \times 10^{3}$ cells per well and cultured to confluence in the growth medium. The cells were scraped away vertically by pipette tip and then incubated for 18 hours in $500 \mu \mathrm{l}$ of serum-free EGM-2 medium with $5 \mathrm{ng}$ of rhVEGF or $4 \times 10^{5}$ numbers of empty and VEGF polyhedra. For endostatin polyhedra $(\mathrm{H} 1 /$ Endo polyhedra and Endo/VP3), $4 \times 10^{5}$ numbers of polyhedra were added to $500 \mu \mathrm{l}$ of serum-free EGM-2 medium containing rhVEGF $(10 \mathrm{ng} / \mathrm{ml})$.

Fig. 3 Stimulation and inhibition of network and tube formation of HUVECs by VEGF and endostatin polyhedra. (A) Subconfluent culture of HUVECs was suspended in the starvation 
medium to synchronize cell cycles. After 2 ng of rhVEGF or $1.6 \times 10^{5}$ numbers of empty and VEGF polyhedra were mixed with ECMatrix solution, aliquots were added to each well of the

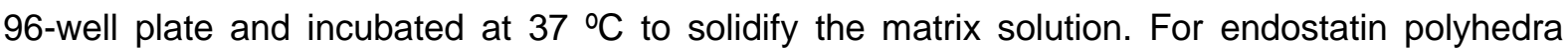
(H1/Endo and Endo/VP3), $1.6 \times 10^{5}$ numbers of polyhedra were added to ECMatrix solution containing rhVEGF $(10 \mathrm{ng} / \mathrm{ml})$. HUVECs $\left(1 \times 10^{4} /\right.$ well $)$ were seeded onto presolidified ECMatrix in each well. Calcein AM solution was added to each well. a, control; b, empty polyhedra; c, rhVEGF; d, VEGF polyhedra; e, H1/Endo polyhedra; f, Endo/VP3 polyhedra. (B) Network formation was photographed using an inverted microscope and tube length of each cell was measured using an image analyzer (ESPEC TECHNO CORP). The graph shows mean +/- S.E. (C) Normal human dermal fibroblast cells were plated into a collagen-coated 24 -well plate at a density of $2 \times 10^{4}$ cells per well and incubated with $500 \mu$ l of Medium 106 supplemented with Low Serum Growth Supplement. The medium was replaced with $500 \mu \mathrm{l}$ of the starvation medium and then $5 \mathrm{ng}$ of rhVEGF or $4 \times 10^{5}$ numbers of empty and VEGF polyhedra were added to each well. For endostatin polyhedra (H1/Endo and Endo/VP3), $4 \times 10^{5}$ numbers of polyhedra were added to $500 \mu \mathrm{l}$ of the starvation medium containing rhVEGF $(10 \mathrm{ng} / \mathrm{ml})$. HUVECs $\left(2 \times 10^{4} /\right.$ well $)$ were seeded into each well and incubated with the assay medium for 11 days at $37^{\circ} \mathrm{C}$. The assay medium with or without rhVEGF $(10 \mathrm{ng} / \mathrm{ml})$ was used for wells of rhVEGF and polyhedra, respectively, at each 2 days medium change. To detect tube formation of HUVECs, mouse anti-human CD31 antibody was employed followed by goat anti-mouse IgG AP conjugate antibody. Tubes were observed by addition of substrate BCIP/NBT. a, control; b, empty polyhedra; c, rhVEGF; d, VEGF polyhedra; e, H1/Endo polyhedra; f, Endo/VP3 polyhedra.

Fig. 4 Inhibition of growth of subcutaneous SCC-VII tumors by intratumoral injection of endostatin polyhedra. Four treatment groups were established: H1/Endo polyhedra, Endo/VP3 polyhedra, empty polyhedra and no treatment as control. Each polyhedron was administrated at 2 and 4 weeks after SCC-VII tumor-cell inoculation and tumor volume was measured at 6 weeks after the 
tumor-cell inoculation. The first quartile and third quartile were indicated by horizontal bars at the bottom and top of the box, respectively. Median is indicated as the center line inside the box. Outliers are indicated by dots.

Fig. 5 Endostatin polyhedra inhibit angiogenesis but not lymphangiogenesis in SCC-VII tumors. (A) Representative double immunofluorescent staining image for CD31, the marker of vascular endothelial cells (green), and LYVE-1, the marker of lymphatic endothelial cells (red). a, control; b, empty polyhedra; c, H1/Endo polyhedra; d, Endo/VP3 polyhedra. (B) CD31-positive endothelial cells were randomly counted at 100-power magnification from five different fields. CD31-positive vessels in treated tumors treated with endostatin polyhedra $(\mathrm{H} 1 /$ Endo polyhedra and Endo/VP3 polyhedra) were markedly lower than for control and empty polyhedra groups $\left({ }^{\star} \mathrm{P}<0.05,{ }^{* \star} \mathrm{P}<\right.$ 0.01). Vessel numbers were compared using Student's $t$ test. The graph shows mean +/- S.E. (C) Quantification of LYVE-1-positive lymphatic vessels in the tumor tissues. There was no significant difference between the control, empty polyhedron, and endostatin polyhedra groups (H1/Endo polyhedra and Endo/VP3 polyhedra). Vessel numbers were compared using Student's t test. The graph shows mean $+/-$ S.E. 
A Without PDI

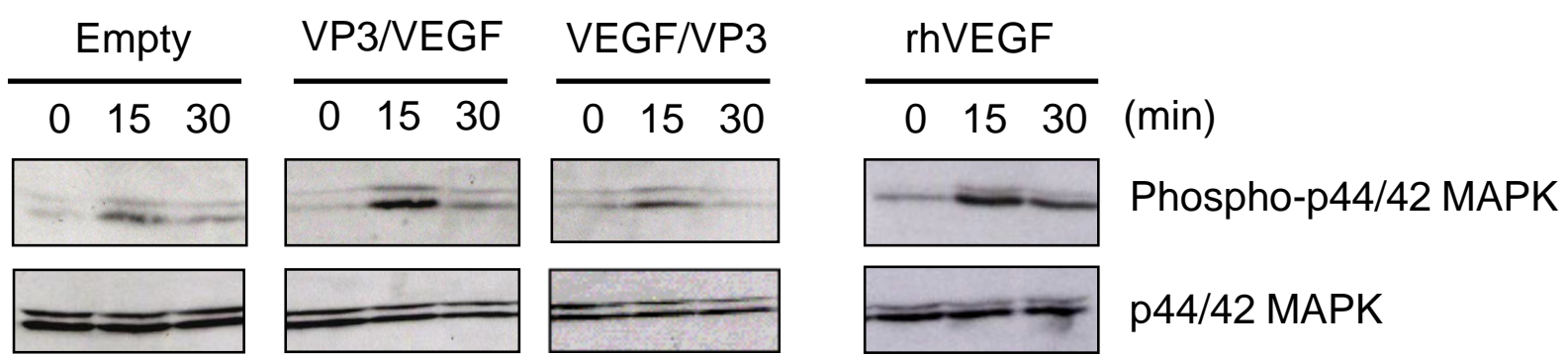

With PDI

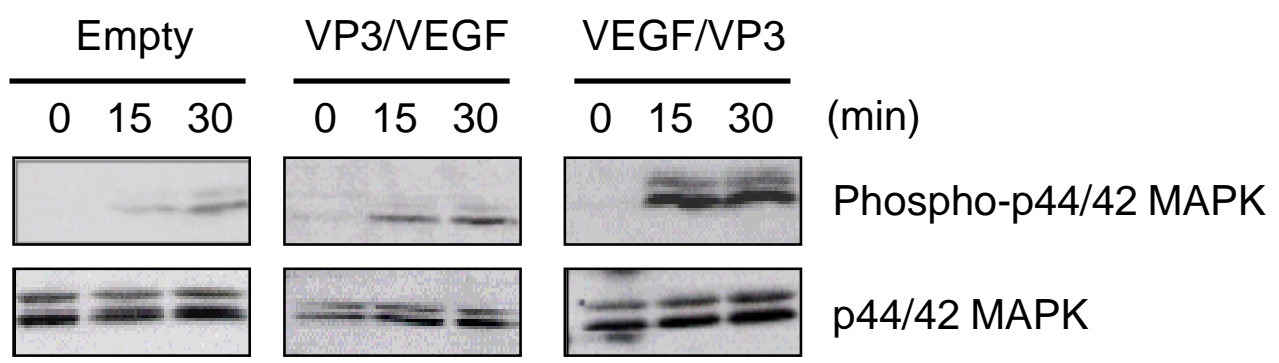

B

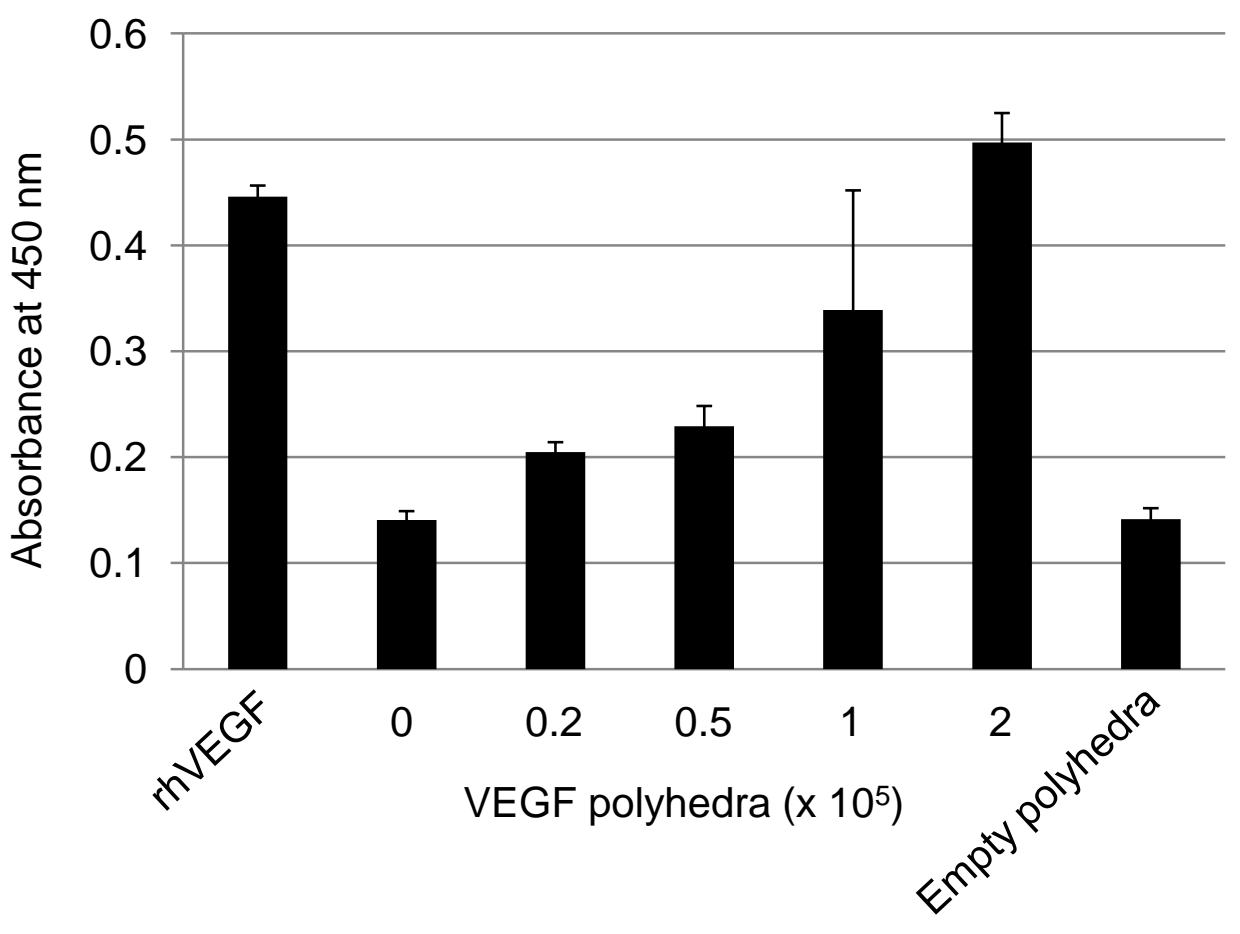

Fig.1 

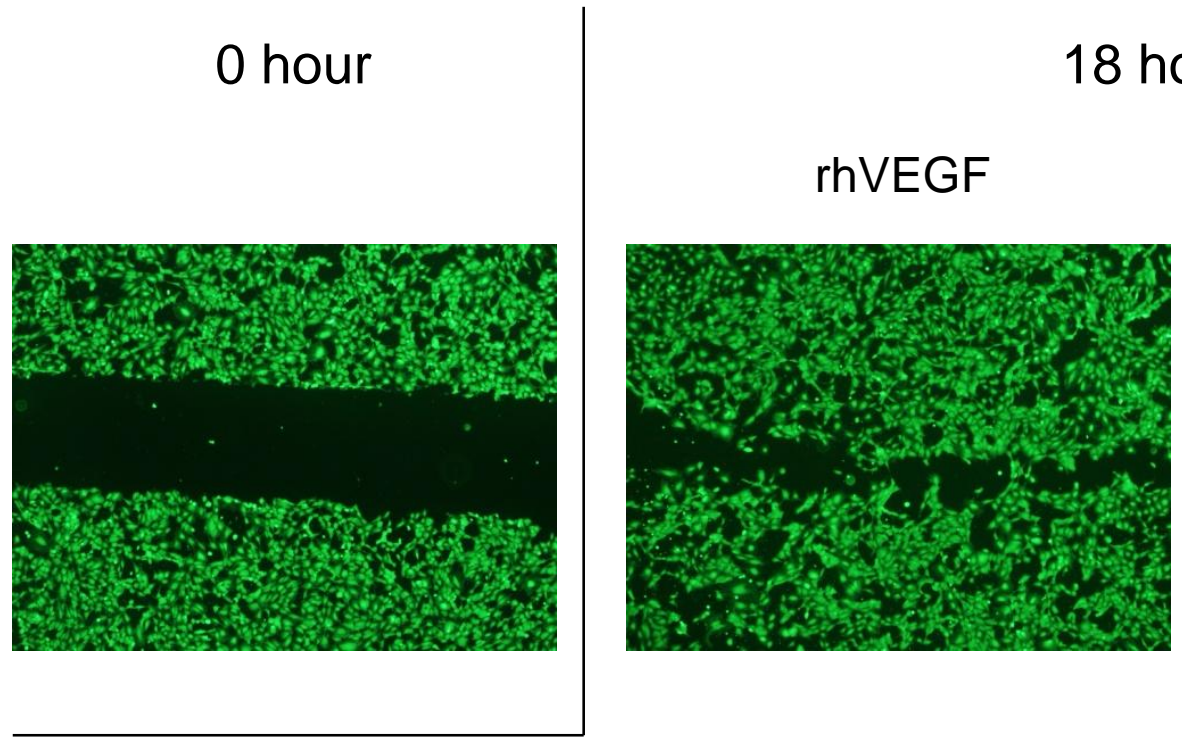

18 hours

rhVEGF

VEGF polyhedra

Empty polyhedra

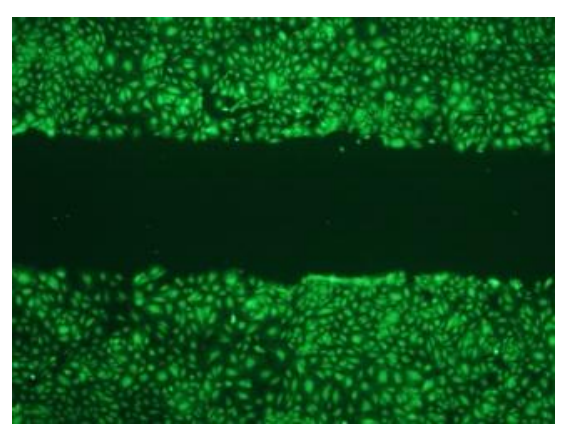

H1/Endo polyhedra

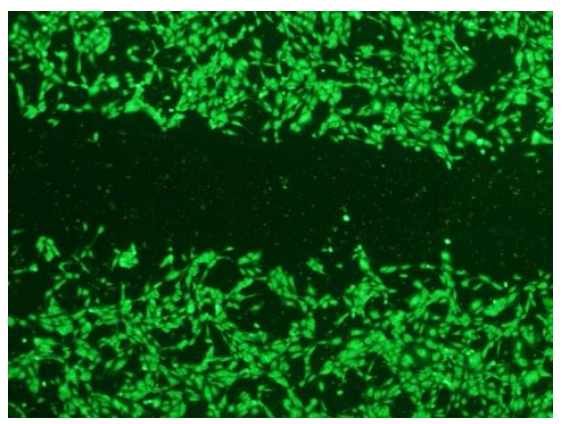

Endo/VP3 polyhedra

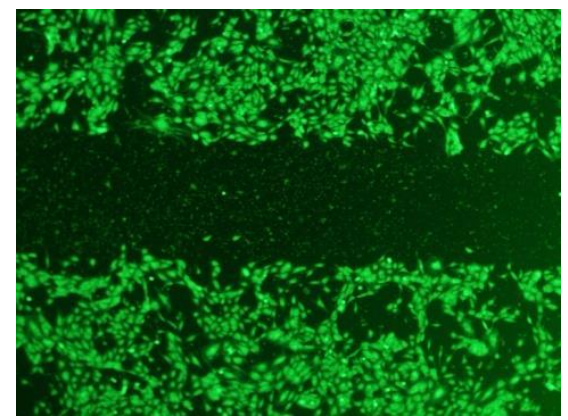

Fig.2 


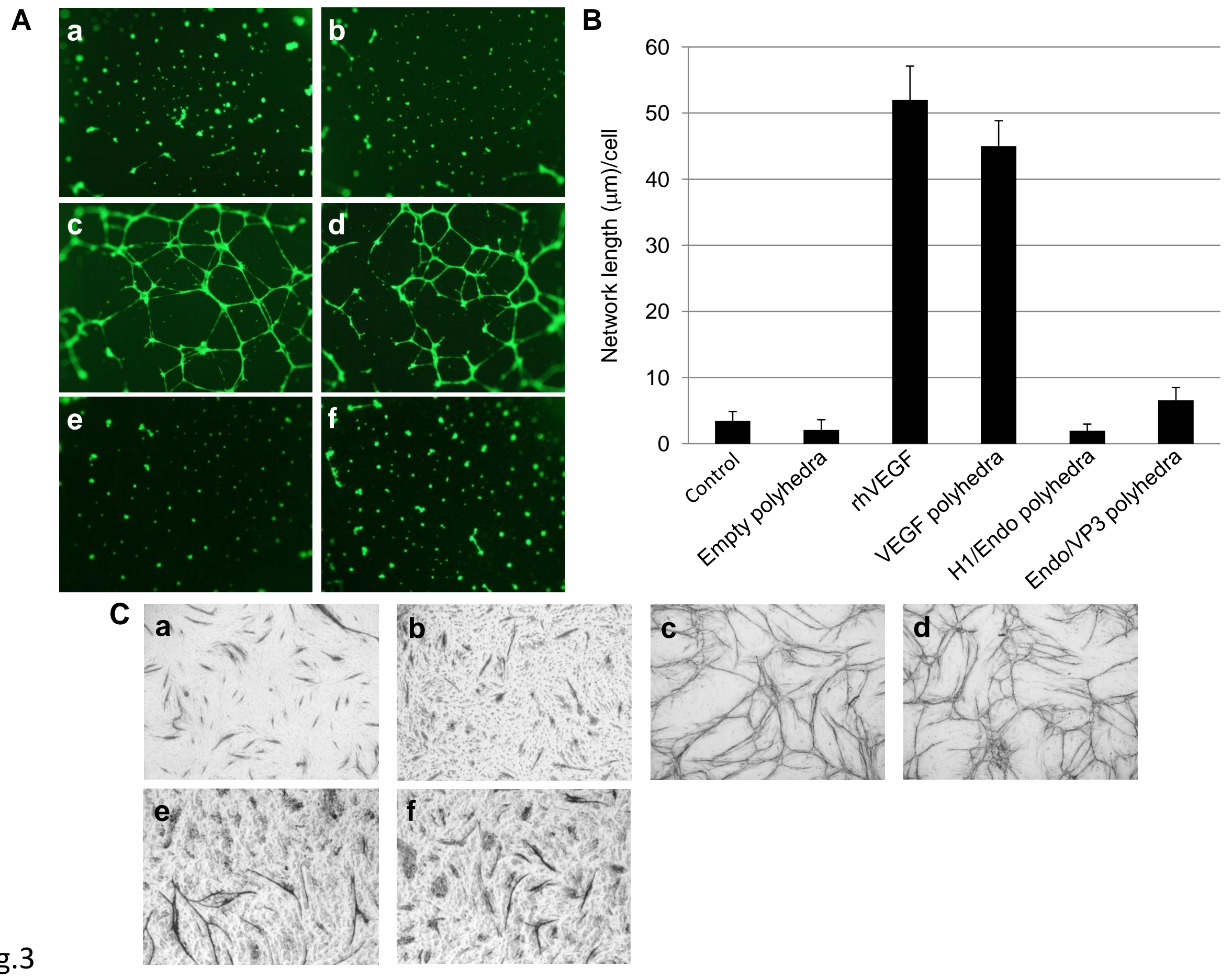

Fig.3 


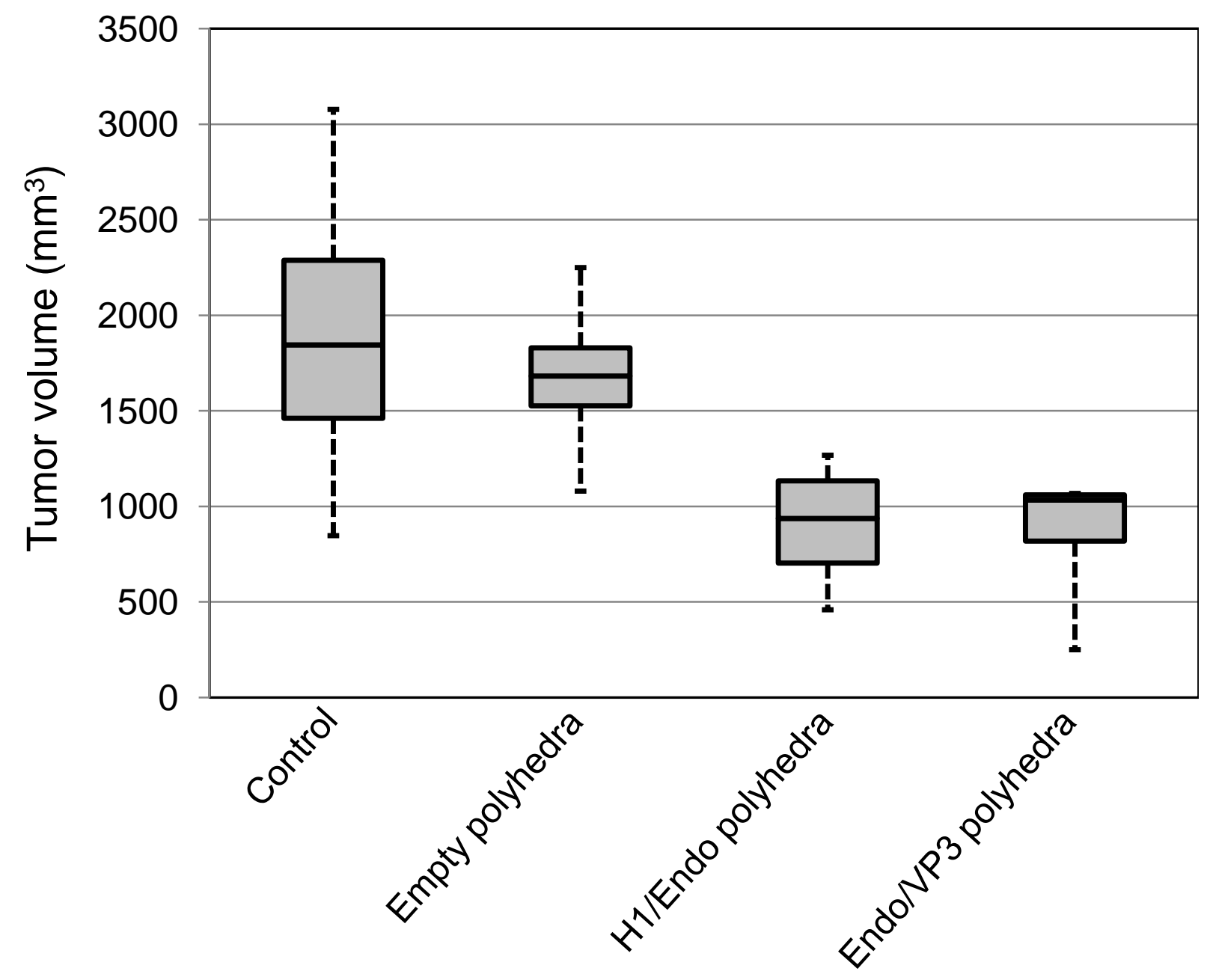

Fig.4 
A

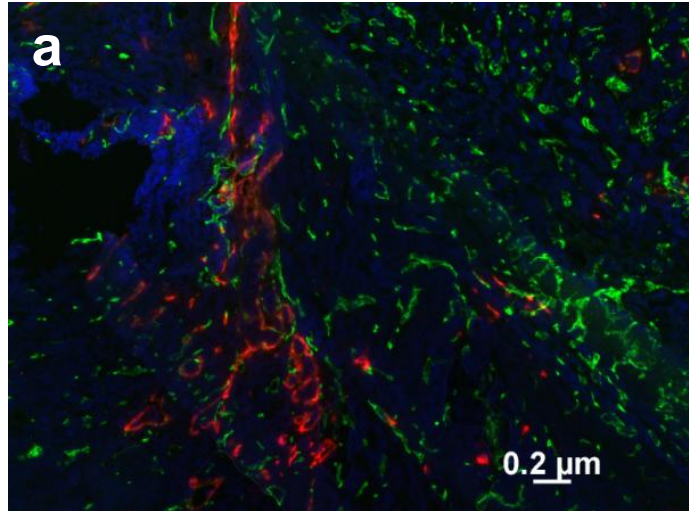

c
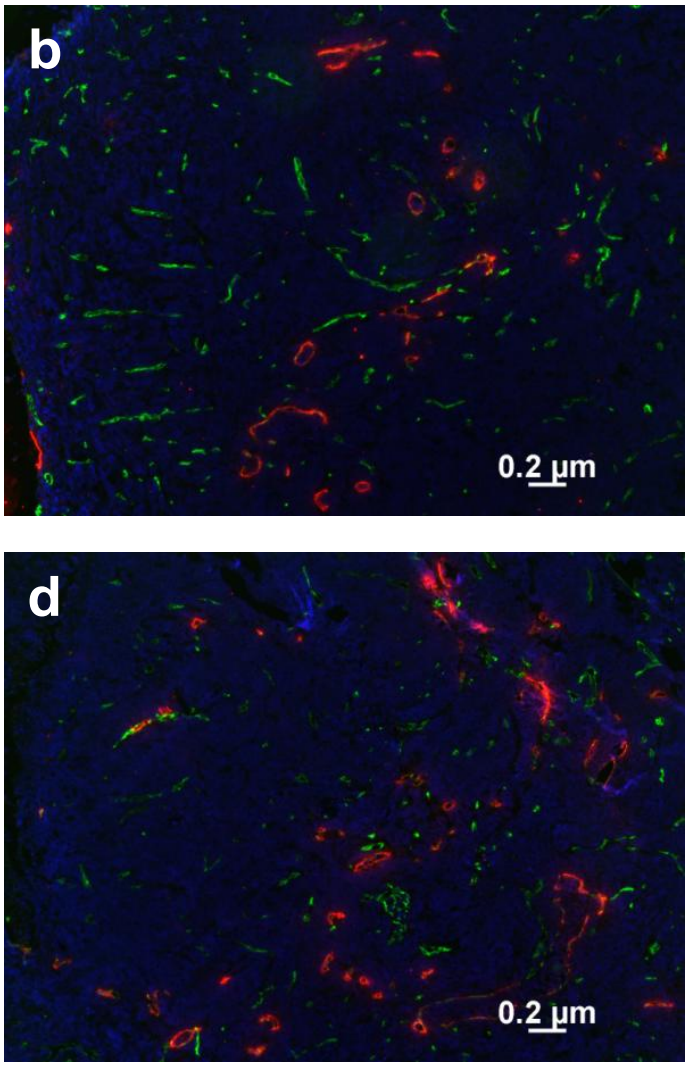

B

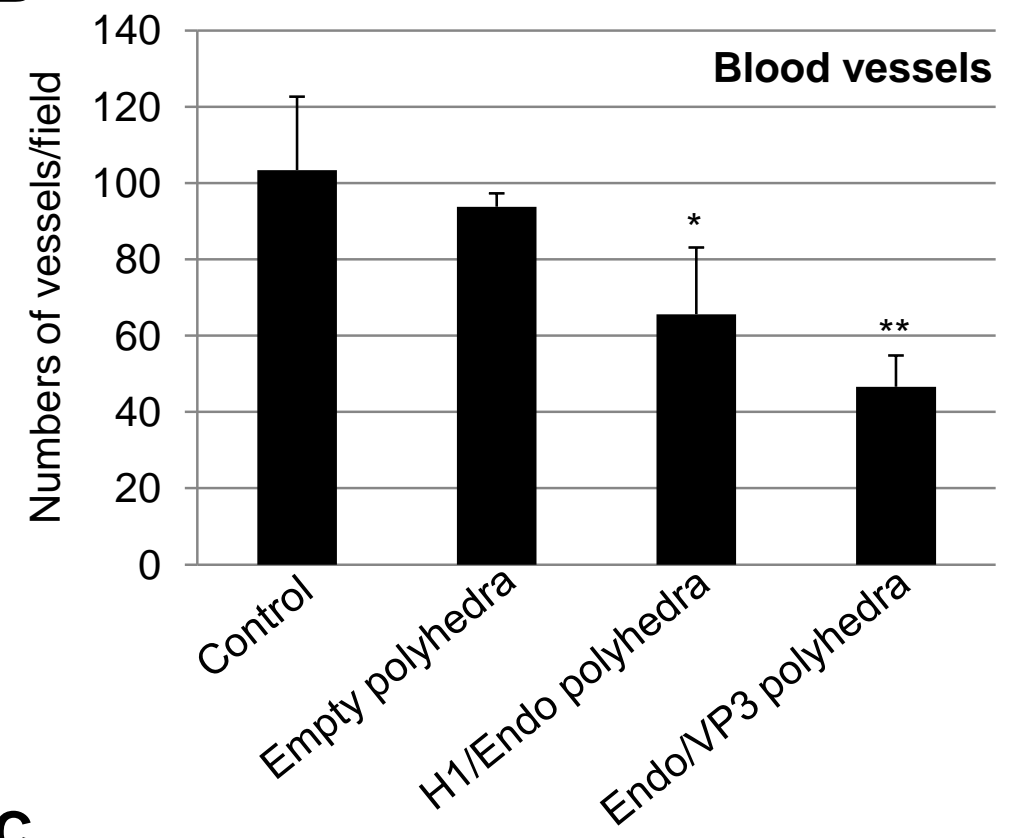

C

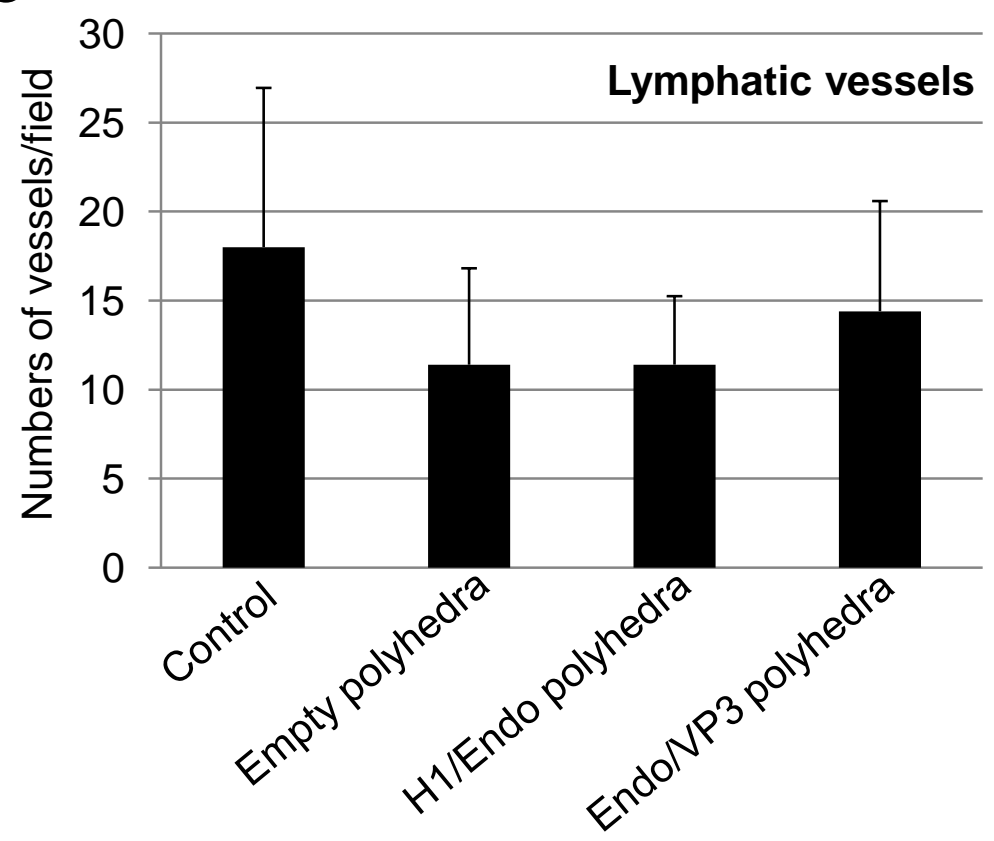

Fig.5 